\title{
Editorial
}

\section{Information and Modeling in Complexity}

\author{
Carlo Cattani, ${ }^{1}$ Shengyong Chen, ${ }^{2}$ and Gani Aldashev ${ }^{3}$ \\ ${ }^{1}$ Department of Mathematics, University of Salerno, Via Ponte Don Melillo, 84084 Fisciano, Italy \\ ${ }^{2}$ Department of Computer Science, Zhejiang University of Technology, 310023 Hangzhou, China \\ ${ }^{3}$ Department of Economics, University of Namur, Rempart de la Vierge 8, 5000 Namur, Belgium
}

Correspondence should be addressed to Carlo Cattani, ccattani@unisa.it

Received 13 February 2012; Accepted 13 February 2012

Copyright (c) 2012 Carlo Cattani et al. This is an open access article distributed under the Creative Commons Attribution License, which permits unrestricted use, distribution, and reproduction in any medium, provided the original work is properly cited.

\section{Overview of the Issue}

Current research in engineering systems builds theories represented by mathematical models that aim at understanding fundamental questions of systems' spatial structure, self-organization, environmental interaction, behaviour, and development. Thus, engineers are increasingly facing the challenge of dealing with complexity in advanced engineering applications that are based on efficient mathematical models.

The topics that deal with these issues can be divided into two large parts: (i) development of general mathematical methods/models, and (ii) specific applications in particular domains.

The focus of this issue on topics of information and modeling complexity is both on the mathematical models and the engineering applications in complex systems. Moreover, we are interested in both new theoretical developments and the studies of practical implementation concerning modeling, complexity, fractals, statistics, and signal extraction and transformation.

The complexity theory is the analysis of complex systems, which can be approached from many different points of view. Mathematically it refers to nonlinear dynamical systems which are very sensitive to initial conditions, in a such way that a small perturbation of these could have unpredictable consequences on the evolution equations. Moreover the future behaviour or long-term prediction of the system cannot be fully determined, so that the uniqueness of the solution fails. Complex systems were also related to chaotic systems and to chaos dynamics. Common sense reasoning might lead one to believe that complexity and chaos should be considered as the same characteristic of the state of disorder. However, these two concepts should not be confused, since they refer to different questions. A chaotic system 
is a disordered state which follows from the strong dependence on initial conditions, from the existence of some attractors and dense periodic orbits. The strong dependence on initialboundary conditions means that a small variation thereof, from one initial state to another very close, could lead to some different future orbits. In other words, the time evolution for two very close states is divergent in future. Sometime a state of disorder can be also considered as a random state. In other words, one can hardly understand from observed experimental data if they come from the evolution of a chaotic system or if they are simply a collection of random values. Frequency analysis, wavelet analysis, power spectrum method, information theory, entropy, and fractal dimension are expedient tools which enable us to analyze data trying to single out some deterministic time series by removing what can be considered a pure random process.

A key issue in all fields of complexity is the signal extraction and processing. In fact, signals extract such information from the complex phenomena being measured; therefore, the signal analysis can be used to study the complex phenomena. However, a main difficulty comes from the fact that signals are typically some time series having both a regular and a random component. Separating these two components is the essence of signal extraction problem.

Randomness, multifractality and chaos are also epitomizing features of complexity. A complex system can be also considered as a chaotic system, in the sense that it can be sensitive to initial conditions, that its evolution could be also disturbed by some random noise and that it shows some noninteger dimensions typical of fractals. However, a complex system is characterized by some more interesting features, such as a multiparametric dependence or self-organizing multiscale activity. Indeed, being a multidisciplinary topic, complexity does not have a unique definition. We can roughly define complexity as one of the following three:

(1) scale transition from a micro- to macroscale, such that a disordered set of individuals behaves as sole entity;

(2) the analysis of a unique entity resulting from the interaction of many parts;

(3) transition from disorder to organization.

It is clear that a special feature of complex system is the organization or selforganization arising from a chaotic-random state. However, there are still some open debates on a clear definition of organization. We can assume for organization the meaning of predictable, in the sense that there exists a dynamical system able to describe the evolution of the complex system when it is organized. The transition from disorder to organization is still unclear. Many models propose the emergence of order as the scale transition from the microscale, where each individual is characterized by its own activity, to a macroscale where more individuals agree on a unique strategy, which could be different from the usual activity at the microscale.

Self-organizing complex systems are often/essentially maintained by information flows, thus implying the quantification of information. Fundamental tools of signal processing have been recently developed from the elementary topics of compression data, storing and communicating data into more broad applications of information theory such as neurobiology, Internet networks, language processing, quantum computing, data mining, and many more. Any information flow is characterized by a measure, originally given by Shannon, related to uncertainty and disorder in the flow. Shannon entropy is the most popular tool to give a measure of the uncertainty in predicting the evolution of a stochastic variable. More outcomes have a variable, the higher is the entropy, so that this measure can 
be also taken as the measure of randomness: the higher is the entropy, the more random is the variable evolution. Many alternative definitions of the Shannon entropy have been recently proposed starting from the Kolmogorov-Sinai entropy, Rényi entropy, topological entropy, and so forth.

The theory of complexity applied to advanced engineering systems requires subtle and efficient mathematical models obtained by developing classical tools for complex systems. The increasing demand for engineering systems able to handle complex phenomena is nowadays pushing scholar's investigations in all directions of knowledge, such as medical biological and visual systems, environment, optimisation, structural complexity, advanced composite material, vibrations, social science, economy, Internet architecture, and processing in networks.

The analysis of general principles that lie behind these problems in so many different fields, transversally across different specific applications is the area of highly active research. Solutions of the arising mathematical problems attempt to map general principles for modeling how the complex systems operate. This area is a burgeoning field of research due to both the practical significance of the applications and the general scientific importance.

From the current states and trends in the issue and related topics of information and modelling in complexity, although some solutions and models become available, most problems remains open and research is highly active in this field. In the near future, we expect many more contributions that will address all of the key aspects raised above, in particular, the development of general mathematical methods and models, analyses of practical signalextraction problems, and applications in the study of self-organizing problems in social sciences.

Carlo Cattani

Shengyong Chen

Gani Aldashev 


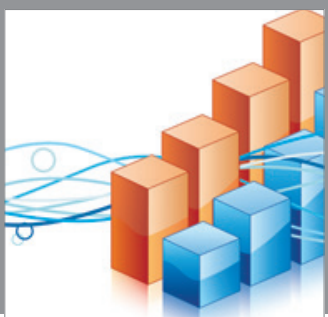

Advances in

Operations Research

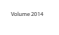

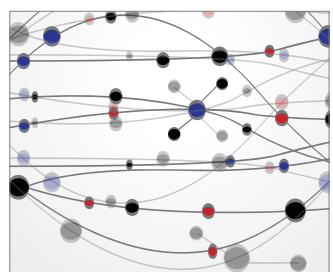

\section{The Scientific} World Journal
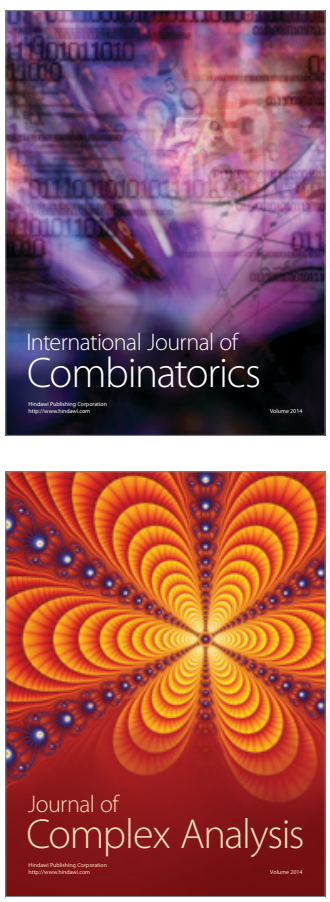

International Journal of

Mathematics and

Mathematical

Sciences
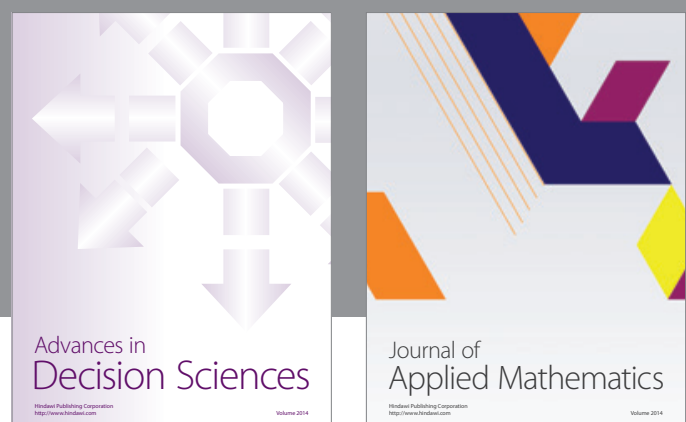

Journal of

Applied Mathematics
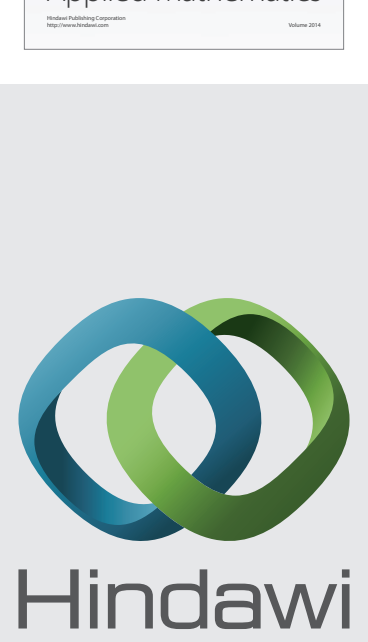

Submit your manuscripts at http://www.hindawi.com
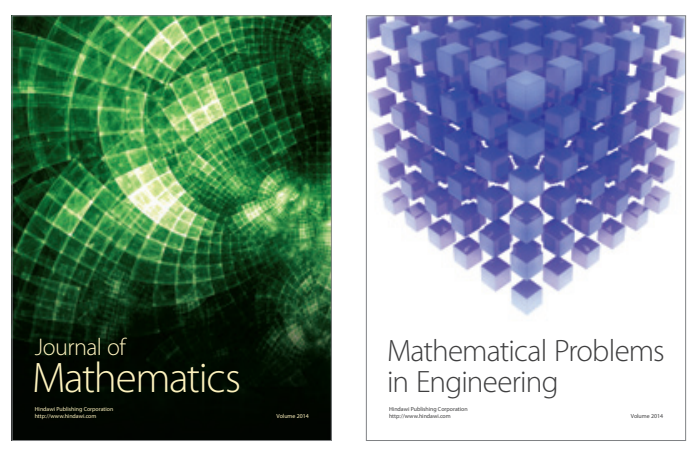

Mathematical Problems in Engineering
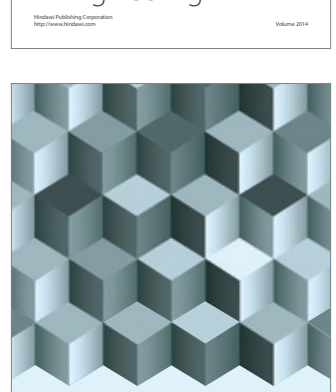

Journal of

Function Spaces
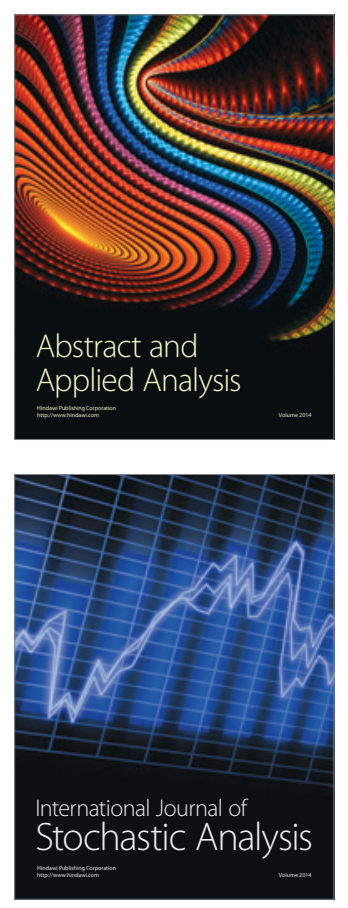

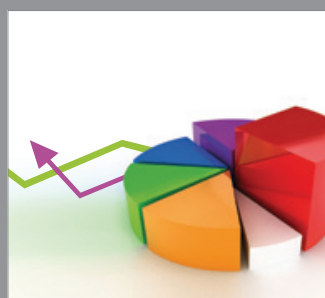

ournal of

Probability and Statistics

Promensencen
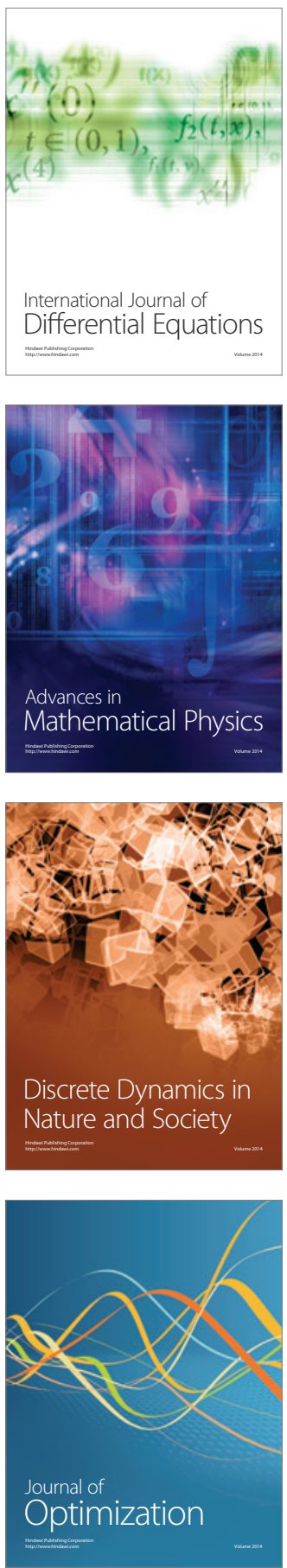